\title{
FREE QUASICONFORMALITY IN BANACH SPACES III
}

\author{
Jussi Väisälä \\ Helsingin yliopisto, Matematiikan laitos \\ Hallituskatu 15, SF-00100 Helsinki, Finland
}

\begin{abstract}
The paper deals with freely quasiconformal and coarsely quasihyperbolic maps between domains in Banach spaces. New characterizations for the free quasiconformality are given. The correspondence of the boundary components is studied.
\end{abstract}

\section{Introduction}

This paper is continuation to [Vä $\left.{ }_{1}\right]$ and $\left[V \ddot{a}_{2}\right]$. We assume that the reader is familiar with these papers. They will be cited as I and II. For example, I.2.5 means the result 2.5 of [ $\left[\ddot{a}_{1}\right]$.

We shall use the notation and terminology of I and II. In particular, $E$ and $E^{\prime}$ will be real Banach spaces of dimension at least 2, and $G \subset E$ and $G^{\prime} \subset E^{\prime}$ will be domains. Whenever a statement involves the quasihyperbolic metric $k=k_{G}$ of $G$ or the distance $\delta(x)=d(x, \partial G)$, we shall tacitly assume that $G \neq E$.

Suppose that $f: G \rightarrow G^{\prime}$ is a homeomorphism with $G \neq E, G^{\prime} \neq E^{\prime}$. In II.4.14 we gave the following quantitative implications:

$$
M-\mathrm{QH} \Rightarrow \varphi \text {-FQC } \Rightarrow \varphi \text {-solid } \Rightarrow(M, C)-\mathrm{CQH} .
$$

Here $M \geq 1, C \geq 0$, and $\varphi:[0, \infty) \rightarrow[0, \infty)$ is a homeomorphism with $\varphi(t) \geq t$. We recall that $\mathrm{QH}=$ quasihyperbolic, $\mathrm{FQC}=$ freely quasiconformal and $\mathrm{CQH}=$ coarsely quasihyperbolic. The main object of this paper will be the class of $\mathrm{CQH}$ maps. We recall that a homeomorphism $f: G \rightarrow G^{\prime}$ is $(M, C)$-CQH if

$$
(k(x, y)-C) / M \leq k^{\prime}(f x, f y) \leq M k(x, y)+C
$$

for all $x, y \in G$, where $k=k_{G}$ and $k^{\prime}=k_{G^{\prime}}$. The map $f$ is $M-\mathrm{QH}$ if it is $(M, 0)$-CQH. The definitions of the other classes were given in I.3.4, and they will be recalled in 2.18 .

In Section 2 we analyze the concept of $\mathrm{CQH}$ maps and also obtain new characterizations for FQC maps. Section 3 deals with the boundary correspondence and the cluster sets of CQH maps. The difference between the cases $\operatorname{dim} E<\infty$ and $\operatorname{dim} E=\infty$ is essential but less striking than in the case of arbitrary homeomorphisms. An example in a function space is given in Section 4.

I thank again Pekka Alestalo, Jouni Luukkainen and Olli Martio for useful discussions.

1991 Mathematics Subject Classification: Primary 30C65; Secondary 46B20. 


\section{CQH maps}

2.1. Introduction to Section 2. In I.3.8 we characterized the solid maps in terms of relativity. It turns out that the corresponding result for $\mathrm{CQH}$ maps is false in general but true for a large class of domains, called natural domains. All finite-dimensional domains and all uniform domains are natural. We also show that quasimöbius maps relative to the boundary of natural domains are $\mathrm{CQH}$. We next show that fully $(M, C)-\mathrm{CQH}$ maps are precisely the FQC maps and also obtain the following simple geometric characterization for FQC maps: There is $M>0$ such that

$$
d(f \bar{B}(x, r)) \leq M d(f \bar{B}(x, r), \partial f B(x, 2 r))
$$

for each $x \in G$ and $r \leq d(x, \partial G) / 2$ and such that $f^{-1}$ satisfies the same condition.

We recall from II.6.8 that the relative distance of points $x, y \in G \neq E$ is

$$
r_{G}(x, y)=\frac{|x-y|}{\delta(x) \wedge \delta(y)},
$$

where as always $\delta(x)=d(x, \partial G)$.

Each domain $G \neq E$ with its QH metric $k$ is unbounded and $c$-quasiconvex for each $c>1$. Hence II.4.8 gives almost directly the following result:

2.2. Theorem. For a homeomorphism $f: G \rightarrow G^{\prime}$, the following conditions are quantitatively equivalent:

(1) $f$ is $(M, C)-\mathrm{CQH}$.

(2) There are $t_{1} \geq 0$ and $M_{1} \geq 1$ such that

$$
k(x, y) / M_{1} \leq k^{\prime}(f x, f y) \leq M_{1} k(x, y)
$$

whenever $x, y \in G$ and $k(x, y) \geq t_{1}$.

(3) There are $t_{0}>0$ and $M_{0}>0$ such that

$$
\begin{aligned}
k(x, y) & \leq t_{0} \text { implies } k^{\prime}(f x, f y) \leq M_{0} \\
k^{\prime}(f x, f y) & \leq t_{0} \text { implies } k(x, y) \leq M_{0} .
\end{aligned}
$$

Proof. Since each domain is unbounded and $c$-quasiconvex in the QH metric for every $c>1$, the equivalence of (1) and (3) follows readily from II.4.8. Furthermore, (1) implies (2) with $t_{1}=2 C, M_{1}=2 M$. Finally assume that (2) holds. If $k^{\prime}(f x, f y) \geq t_{1}$, then $k(x, y) \leq t_{1}$ or $k(x, y) / M_{1} \leq k^{\prime}(f x, f y)$. In both cases $k(x, y) \leq M_{1} k^{\prime}(f x, f y)$. Hence both $f$ and $f^{-1}$ satisfy the condition (2) of II.4.8 in the QH metric. Consequently, they are $C$-coarsely $M$-Lipschitz with $(M, C)$ depending on $\left(M_{1}, t_{1}\right)$ and we obtain (1). $\square$ 
2.3. Theorem. Suppose that a map $f: G \rightarrow G^{\prime}$ is $C$-coarsely $M$-Lipschitz in the QH metric. Then there is an increasing function $\theta:[0,1) \rightarrow[0, \infty)$, depending only on $(M, C)$, such that

$$
\frac{|f x-f y|}{\delta^{\prime}(f x)} \leq r_{G^{\prime}}(f x, f y) \leq \theta\left(\frac{|x-y|}{\delta(x)}\right)
$$

whenever $|x-y|<\delta(x)$, and

$$
r_{G^{\prime}}(f x, f y) \leq \theta\left(r_{G}(x, y)\right)
$$

whenever $r_{G}(x, y)<1$.

Proof. Observe that $\theta(t)$ is not required to tend to zero as $t \rightarrow 0$. Assume that $|y-x|=t \delta(x)$ with $0 \leq t<1$. Then I.2.2 implies $k(x, y) \leq t /(1-t)$ and

$$
\log r_{G^{\prime}}(f x, f y) \leq k^{\prime}(f x, f y) \leq M k(x, y)+C \leq M t /(1-t)+C .
$$

This proves (1), and (2) is a direct corollary. $\square$

2.4. Example. If $f: G \rightarrow G^{\prime}$ is an $(M, C)$-CQH homeomorphism, both $f$ and $f^{-1}$ satisfy the conditions of 2.3. The converse is not true as is seen from the following example; cf. I.4.12.

Let $E$ be a Hilbert space with an orthonormal base $\left(e_{j}\right)_{j \in \mathbf{Z}}$. We consider the broken line

$$
A=\cup\left\{\left[e_{j-1}, e_{j}\right]: j \in \mathbf{Z}\right\},
$$

and for $r=1 / 20$, the broken tubes

$$
D=B(A, r), G=B(A, 2 r) .
$$

Suppose that $f: G \rightarrow G$ is a homeomorphism with $f \mid G \backslash D=$ id. Since $f \mid A$ is rather arbitrary, $f$ need not be CQH. However, we show that it satisfies the condition (1) of 2.3 .

Write $M=d(G)<\infty$ and observe that $\delta(x) \geq r$ for $x \in D$. Suppose that $x, y \in G$ with $|x-y|=t \delta(x), 0<t<1$. If $x \in D$, then

$$
\frac{|f x-f y|}{\delta^{\prime}(f x)} \leq \frac{M}{r}
$$

If $x \notin D$ and $y \in D$, then $\delta(x) \geq \delta(y)-|y-x| \geq r-t \delta(x)$, which implies

$$
\delta^{\prime}(f x)=\delta(x) \geq \frac{r}{1+t},
$$


and hence

$$
\frac{|f x-f y|}{\delta^{\prime}(f x)} \leq \frac{M(1+t)}{r} .
$$

Finally, if $x \notin D$ and $y \notin D$, then

$$
\frac{|f x-f y|}{\delta^{\prime}(f x)}=\frac{|x-y|}{\delta(x)}=t .
$$

Hence the condition (1) of 2.3 is true with the constant function $\theta(t)=2 M / r$. Of course, $f^{-1}$ satisfies the same condition.

We next introduce the class of natural domains and prove in 2.10 a converse of 2.3 for these domains.

2.5. Natural domains. Suppose that $\emptyset \neq A \subset G \neq E$. We write

$$
r_{G}(A)=\sup \left\{r_{G}(x, y): x \in A, y \in A\right\}, \quad \bar{r}_{G}(A)=\frac{d(A)}{d(A, \partial G)} .
$$

The number $\bar{r}_{G}(A)$ was written as $r_{G}(A)$ and called the relative size of $A$ in [TV]. However, these numbers are not essentially different, since we always have

$$
r_{G}(A) \leq \bar{r}_{G}(A) \leq 2 r_{G}(A) .
$$

The first inequality is clear. To prove the second one, let $a, b, c \in A$. Then

$$
\frac{|a-b|}{\delta(c)} \leq \frac{|a-c|}{\delta(c)}+\frac{|c-b|}{\delta(c)} \leq r_{G}(a, c)+r_{G}(c, b) \leq 2 r_{G}(A),
$$

and hence $\bar{r}_{G}(A) \leq 2 r_{G}(A)$.

Let $\psi:[0, \infty) \rightarrow[0, \infty)$ be an increasing function, but we do not require that $\psi(0)=0$. We say that a domain $G \neq E$ is $\psi$-natural if either $G=E$ or

$$
k_{G}(A) \leq \psi\left(r_{G}(A)\right)
$$

for every nonempty connected set $A \subset G$ with $r_{G}(A)<\infty$. Here $k_{G}(A)$ is the QH diameter of $A$. A domain is called natural if it is $\psi$-natural for some $\psi$.

The broken tube $G$ of 2.4 is not a natural domain, since we have $k_{G}(A)=\infty$, $r_{G}(A) \leq M / 2 r<\infty$ for the broken line $A$. However, the next results show that the class of natural domains is fairly large:

2.7. Theorem. Suppose that $\operatorname{dim} E=n<\infty$. Then each domain in $E$ is $\psi$-natural with $\psi$ depending only on $n$.

Proof. There is a $\sqrt{n}$-bilipschitz map of $E$ onto $R^{n}$; cf. I.6.1. Hence we may assume that $E=R^{n}$. The result follows from [TV, 6.9] or from [Vu, 2.18]. व 
2.8. Theorem. $A \mathrm{QH} \psi$-uniform domain is $\psi$-natural. $A c$-uniform domain is $\psi$-natural with $\psi=\psi_{c}$.

Proof. The first part follows directly from the definition of QH $\psi$-uniformity in II.6.8. The second part follows from II.6.16. $\square$

2.9. Corollary. A convex domain is $\psi$-natural with $\psi(t)=t$. $\square$

2.10. Theorem. Suppose that $G \neq E, G^{\prime} \neq E^{\prime}$, that $G^{\prime}$ is $\psi$-natural and that $f: G \rightarrow G^{\prime}$ is a continuous map. Suppose also that $0<t_{0}<1, M_{0} \geq 0$, and that

$$
|f x-f y| \leq M_{0} \delta^{\prime}(f x)
$$

whenever $x, y \in G$ and $|x-y| \leq t_{0} \delta(x)$. Then $f$ is $C$-coarsely $M$-Lipschitz in the QH metrics of $G$ and $G^{\prime}$. Moreover, $M$ and $C$ depend only on $v=\left(t_{0}, M_{0}, \psi\right)$.

Proof. By II.4.8 it suffices to find $M=M(v)$ such that $k(a, b) \leq t_{0} / 6$ implies $k^{\prime}(f a, f b) \leq M$. Assume that $a, b \in G$ with $k(a, b) \leq t_{0} / 6$ and write $A=\bar{B}\left(a, t_{0} \delta(a) / 3\right)$. Since $k(a, b)<1$, I.2.5 yields

$$
\frac{|a-b|}{\delta(a)} \leq 2 k(a, b) \leq \frac{t_{0}}{3}
$$

and hence $a, b \in A$. Thus it suffices to find an estimate $k^{\prime}(f A) \leq M$. For each pair $x, y \in A$ we have

$$
\frac{|x-y|}{\delta(x)} \leq \frac{2 t_{0} \delta(a) / 3}{\delta(a)-t_{0} \delta(a) / 3}=\frac{2 t_{0}}{3-t_{0}} \leq t_{0} .
$$

Hence $r_{G^{\prime}}(f x, f y) \leq M_{0}$, which implies $r_{G^{\prime}}(f A) \leq M_{0}$. Since $G^{\prime}$ is $\psi$-natural, this yields $k^{\prime}(f A) \leq \psi\left(M_{0}\right)=M(v)$. व

2.11. Corollary. Suppose that $G \neq E, G^{\prime} \neq E^{\prime}$, that $G$ and $G^{\prime}$ are $\psi$ natural and that $f: G \rightarrow G^{\prime}$ is a homeomorphism. Suppose also that $0<t_{0}<1$, $M_{0}>0$, and that

(1) $|x-y| \leq t_{0} \delta(x)$ implies $|f x-f y| \leq M_{0} \delta^{\prime}(f x)$,

(2) $|f x-f y| \leq t_{0} \delta^{\prime}(f x)$ implies $|x-y| \leq M_{0} \delta(x)$.

Then $f$ is $(M, C)$-CQH with $M, C$ depending only on $v=\left(t_{0}, M_{0}, \psi\right)$. व

2.12. $C Q H$ and relative quasimöbius. Recall from I.5.18 that every QM (= quasimöbius) homeomorphism $f: G \rightarrow G^{\prime}$ is FQC. The converse is true for uniform domains by II.7.16. Analogously, we proved in II.7.9 that a CQH map $f: G \rightarrow G^{\prime}$ between uniform domains can be extended to a homeomorphism $\bar{f}: \bar{G} \rightarrow \bar{G}^{\prime}$, which is $\mathrm{QM}$ rel $\partial G$. The relatively QM maps were defined in II.5.2. It is natural to ask whether QM rel $\partial G$ implies CQH. The answer is negative, as is easily seen from the example in 2.4. In II.7.22 we showed that the answer is positive if the domains $G$ and $G^{\prime}$ are $\mathrm{QH}$ uniform. In 2.17 we shall extend this result to all natural domains and hence to all domains in $R^{n}$. Before that we prove the QM invariance of natural domains. 

Then

2.13. Lemma. Let $u(x)=x /|x|^{2}$, and let $G$ be a domain with $0 \notin G$.

$$
r_{u G}(u a, u b) \leq 18\left(1+r_{G}(a, b)\right)^{2}
$$

for all $a, b \in G$.

Proof. This was proved in Case 1 of the proof of II.6.25. In fact, the constant 18 could be replaced by 9 with a more careful choice of the point $x$. $\square$

2.14. Lemma. Suppose that $f: \bar{G} \rightarrow \bar{G}^{\prime}$ is $\eta$-QM rel $\partial G$ and that $f G=G^{\prime}$. Suppose also that $a, b \in G$ with $r_{G}(a, b) \leq t$. Then $r_{G^{\prime}}(f a, f b) \leq \mu(t, \eta)<\infty$.

Proof. If $f$ is the inversion $u$, the result follows from 2.13. In the general case we use auxiliary inversions and translations to reduce the situation to the case where $\infty \in \partial G$ and $f(\infty)=\infty$. Forgetting the point $\infty, f$ is then $\eta$-QS rel $\partial G$.

We may assume that $\delta^{\prime}(f a) \leq \delta^{\prime}(f b)$. Choose $z \in \partial G$ such that $|f a-f z| \leq$ $2 \delta^{\prime}(f a)$. Then

$$
\begin{aligned}
r_{G^{\prime}}(f a, f b) & =\frac{|f a-f b|}{\delta^{\prime}(f a)} \leq 2\left(1+\frac{|f b-f z|}{|f a-f z|}\right) \\
& \leq 2\left(1+\eta\left(\frac{|b-z|}{|a-z|}\right)\right) \leq 2\left(1+\eta\left(1+\frac{|a-b|}{|a-z|}\right)\right) \\
& \leq 2(1+\eta(1+t)) . \square
\end{aligned}
$$

2.15. Lemma. Suppose that $f: G \rightarrow G^{\prime}$ is an $\eta$-QM homeomorphism and that $G$ is $\psi$-natural. Then $G^{\prime}$ is $\psi_{1}$-natural with $\psi_{1}$ depending only on $\psi$ and $\eta$.

Proof. Let $A^{\prime} \subset G^{\prime}$ be a connected set with $r_{G^{\prime}}\left(A^{\prime}\right) \leq t$, and write $A=$ $f^{-1} A^{\prime}$. Since $f^{-1}$ is $\eta^{\prime}$-QM with $\eta^{\prime}(r)=\eta^{-1}\left(r^{-1}\right)^{-1}$ and since absolute $\eta$-QM implies $\eta$-QM rel $\partial G$ for the extension $\bar{f}: \bar{G} \rightarrow \bar{G}^{\prime}, 2.14$ gives an estimate $r_{G}(A) \leq \mu(t, \eta)$. Hence $k(A) \leq \psi(\mu(t, \eta))$. Now $f$ is $\varphi$-FQC with $\varphi=\varphi_{\eta}$ by I.5.18. Thus

$$
k^{\prime}\left(A^{\prime}\right) \leq \varphi(\psi(\mu(t, \eta)))=\psi_{1}(t) \text {. }
$$

2.16. Question. Is 2.15 true for $\mathrm{QM}$ maps rel $\partial G$ ?

2.17. Theorem. Suppose that $f: \bar{G} \rightarrow \bar{G}^{\prime}$ is $\eta$-QM rel $\partial G$ with $f G=G^{\prime}$ and that $G$ and $G^{\prime}$ are $\psi$-natural. Then $f$ is $(M, C)$-CQH with $M, C$ depending only on $\eta$ and $\psi$.

Proof. Performing auxiliary inversions and translations and using 2.15 we may assume that $\infty \in \partial G$ and $f(\infty)=\infty$. Then $f$ is $\eta$-QS rel $\partial G$. By symmetry and by 2.11 , it suffices to find $M_{0}=M_{0}(\eta, \psi)$ such that

$$
|f x-f y| \leq M_{0} \delta^{\prime}(f x)
$$


whenever $x, y \in G$ with $2|x-y| \leq \delta(x)$.

So assume that $2|x-y| \leq \delta(x)$. Choose $z \in \partial G$ with $|f z-f x| \leq 2 \delta^{\prime}(f x)$. Using the relative $\eta$-quasisymmetry of $f$ we obtain

$$
\begin{aligned}
\frac{|f x-f y|}{\delta^{\prime}(f x)} & \leq 2 \frac{|f x-f y|}{|f z-f x|} \leq 2\left(1+\frac{|f z-f y|}{|f z-f x|}\right) \leq 2\left(1+\eta\left(\frac{|z-y|}{|z-x|}\right)\right) \\
& \leq 2\left(1+\eta\left(1+\frac{|y-x|}{|z-x|}\right)\right) \leq 2(1+\eta(3 / 2))=M_{0} . \square
\end{aligned}
$$

2.18. FQC maps. We recall some terminology of Section 3 of I. Let $\varphi:[0, \infty)$ $\rightarrow[0, \infty)$ be a growth function, that is, a homeomorphism with $\varphi(t) \geq t$. A homeomorphism $f: G \rightarrow G^{\prime}$ is $\varphi$-semisolid if

$$
k^{\prime}(f x, f y) \leq \varphi(k(x, y))
$$

for all $x, y \in G$, and $\varphi$-solid if $f$ and $f^{-1}$ satisfy this condition. For a domain $D \subset G$, we let $f_{D}: D \rightarrow f D$ denote the restriction of $f$. The homeomorphism $f$ has fully a given property if $f_{D}$ has this property for each proper subdomain $D \subset G$. This definition slightly differs from that given in I.3.4, where we asked that also $f$ has this property in the case $G \neq E, G^{\prime} \neq E^{\prime}$. However, it is easy to see that these definitions are equivalent for all classes of maps considered in I and II; only the QHQS maps need a special argument. For example, assume that $G \neq E, G^{\prime} \neq E^{\prime}$, and that $f: G \rightarrow G^{\prime}$ is fully $\varphi$-semisolid. We show that $f$ is $\varphi$-semisolid. Let $a, b \in G$. Choose a sequence $\left(x_{n}\right)$ in $G \backslash\{a, b\}$ such that $\delta\left(x_{n}\right) \rightarrow 0$. Write $D_{n}=G \backslash\left\{x_{n}\right\}$. Then

$$
k^{\prime}(f a, f b) \leq k_{f D_{n}}(f a, f b) \leq \varphi\left(k_{D_{n}}(a, b)\right) \rightarrow \varphi(k(a, b))
$$

as $n \rightarrow \infty$.

A fully $\varphi$-solid homeomorphism is called $\varphi$-FQC. It follows that a $\varphi$-FQC homeomorphism is fully $(M, C)$-CQH with $(M, C)$ depending only on $\varphi$. We shall prove the converse in 2.21 . Simultaneously we shall give a simple explicit characterization for free quasiconformality. We first give the one-sided version:

2.19. Theorem. For a homeomorphism $f: G \rightarrow G^{\prime}$, the following conditions are quantitatively equivalent:

(1) $f$ is fully $\varphi$-semisolid.

(2) $f$ is fully $C$-coarsely $M$-Lipschitz in the QH metric.

(3) There is $M>0$ such that

$$
d(f \bar{B}(x, r)) \leq M d(f \bar{B}(x, r), \partial f B(x, 2 r))
$$

whenever $B(x, 2 r) \subset G$. 
Proof. We show that (1) $\Rightarrow(2) \Rightarrow(3) \Rightarrow(1)$. The implication (1) $\Rightarrow(2)$ follows from II.4.8. Indeed, the proof shows that we can choose an arbitrary $C>0$ and then $M=C / \varphi^{-1}(C)$; cf. II.4.14.

(2) $\Rightarrow(3)$ : Assume that $B(x, 2 r)=D \subset G$ and write $B=B(x, r)$. With the notation of 2.5 we must find $M_{0}=M_{0}(M, C)$ such that

$$
\bar{r}_{f D}(f \bar{B}) \leq M_{0} .
$$

The basic inequalities I.2.2 of the QH metric yield

$$
k_{D}(\bar{B}) \leq 2, \quad k_{f D}(f \bar{B}) \geq \log r_{f D}(f \bar{B}) .
$$

Using these, (2), and (2.6) we obtain (2.20) with $M_{0}=2 e^{2 M+C}$.

(3) $\Rightarrow(1)$ : It suffices to show that $f$ is $\varphi$-semisolid with $\varphi=\varphi_{M}$. By I.3.7, it suffices to show that $|x-y| \leq \delta(x) / 16$ implies

$$
\frac{|f x-f y|}{\delta^{\prime}(f x)} \leq \theta\left(\frac{|x-y|}{\delta(x)}\right)
$$

with some function $\theta=\theta_{M}:[0,1 / 16] \rightarrow[0, \infty)$ such that $\theta(t) \rightarrow 0$ as $t \rightarrow 0$.

Suppose that $x, y \in G$ with $|x-y|=t \delta(x), 0<t \leq 1 / 16$. Let $n$ be the unique integer with $2^{-n-1}<t \leq 2^{-n}$; then $n \geq 4$. For $j=0, \ldots, n$, the balls $D_{j}=B\left(x, 2^{j} t \delta(x)\right)$ lie in $G$. Choose a point $z \in \partial G^{\prime}$ with $|f x-z| \leq 2 \delta^{\prime}(f x)$. The condition (3) implies that $d\left(f \bar{D}_{j}, \partial G^{\prime}\right)>0$ for $j \leq n-1$. Hence for these $j$ we can choose the last point $y_{j}$ of the line segment $[f x, z)$ in $\partial f D_{j}$. Set $\lambda=\left|y_{1}-f x\right|$. Then

$$
\lambda \leq\left|y_{j-1}-f x\right| \leq M\left|y_{j-1}-y_{j}\right|
$$

for all $2 \leq j \leq n-1$. After summation we get

$$
(n-2) \lambda \leq M|f x-z| \leq 2 M \delta^{\prime}(f x) .
$$

On the other hand,

$$
|f x-f y| \leq d\left(f \bar{D}_{0}\right) \leq M d\left(f \bar{D}_{0}, \partial f D_{1}\right) \leq M \lambda .
$$

Hence

$$
\frac{|f x-f y|}{\delta^{\prime}(f x)} \leq \frac{2 M^{2}}{n-2}
$$

Since $2^{-n-1}<t$, we obtain

$$
n+1 \geq \frac{\log (1 / t)}{\log 2}
$$


Here $t \leq 1 / 16$ implies $\log (1 / t) \geq 4 \log 2$, and hence

$$
n-2 \geq \frac{\log (1 / t)}{4 \log 2} .
$$

Combining these inequalities yields the desired estimate

$$
\frac{|f x-f y|}{\delta^{\prime}(f x)} \leq \frac{8 M^{2} \log 2}{\log (1 / t)}
$$

2.21. Corollary. For a homeomorphism $f: G \rightarrow G^{\prime}$, the following conditions are quantitatively equivalent:

(1) $f$ is $\varphi$-FQC,

(2) $f$ is fully $(M, C)-\mathrm{CQH}$,

(3) $f$ and $f^{-1}$ satisfy (3) of 2.19. .

\section{Boundary behavior}

3.1. Introduction to Section 3. Suppose that $f: G \rightarrow E^{\prime}$ is a map. We recall that the closures and boundaries of sets in $E$ are always taken in the extended space $\dot{E}=E \cup\{\infty\}$. The cluster set of $f$ at a point $a \in \partial G$ is defined as

$$
\operatorname{clus}(a, f)=\cap \operatorname{cl} f[U \cap G]
$$

where $U$ runs through all neighborhoods of $a$ in $\dot{E}$. If $a \neq \infty$, it suffices to take the balls $U=B(a, r), r>0$; for $a=\infty$ we can take the sets $U=\complement \bar{B}(r)$ where $\complement$ denotes the complement in $\dot{E}$. Equivalently, a point $y \in \dot{E}^{\prime}$ is in clus $(a, f)$ if and only if there is a sequence $\left(x_{j}\right)$ in $G$ such that $x_{j} \rightarrow a$ and $f x_{j} \rightarrow y$. A point $y$ is an asymptotic value of $f$ at $a$ if there is an endcut $\gamma$ of $G$ at $a$, cf. II.3.6, such that $f x \rightarrow y$ as $x \rightarrow a$ on $\gamma$. We let asy $(a, f)$ denote the set of all asymptotic values of $f$ at $a$. Then obviously

$$
\text { asy }(a, f) \subset \operatorname{clus}(a, f) \text {. }
$$

We shall only consider homeomorphisms $f: G \rightarrow G^{\prime}$. Then clearly

$$
\operatorname{clus}(a, f) \subset \partial G^{\prime}
$$

for all $a \in \partial G$. In the finite-dimensional case the cluster set is never empty since $\dot{E}^{\prime}$ is compact. In the infinite-dimensional case the situation is completely different. For example, using the example of Klee [Kl] it is easy to construct a homeomorphism $f: B(1) \rightarrow B(1)$ of the unit ball in a separable Hilbert space such that $f \mid S(1 / 2)=$ id and such that $f$ interchanges the domains $D_{1}=B(1 / 2)$ and $D_{2}=B(1) \backslash \bar{B}(1 / 2)$. Such a map cannot be CQH, since $k\left(D_{1}\right)<\infty$ and 
$k\left(D_{2}\right)=\infty$. In fact, since $B(1)$ is a uniform domain, a CQH map $f: B(1) \rightarrow B(1)$ extends to a homeomorphism of $\bar{B}(1)$ by II.7.9. On the other hand, we constructed in I.4.12 a QH map of a half space onto a broken tube with clus $(a, f)=\emptyset$ at the points $a=0, \infty$.

We conjecture that if $f$ is $\mathrm{CQH}$, then clus $(a, f) \neq \emptyset$ for some $a \in \partial G$. We show in 3.2 that the conjecture is true if $G$ or $G^{\prime}$ is uniform. Next we consider the correspondence of the boundary components in a CQH map $f: G \rightarrow G^{\prime}$. Let $\nu(G)$ denote the number of components of $\partial G$. In the finite-dimensional case we have $\nu(G)=\nu\left(G^{\prime}\right)$ for topological reasons. We show that $\nu(G)=\nu\left(G^{\prime}\right)$ also in the general case provided that the boundary components can be separated by a set of finite $\mathrm{QH}$ diameter. Without this condition, however, one can find a $\mathrm{QH}$ map $f: G \rightarrow G^{\prime}$ such that $\partial G$ is connected while $\partial G^{\prime}$ is not.

3.2. Theorem. Suppose that $f: G \rightarrow G^{\prime}$ is $\mathrm{CQH}$ and that $G^{\prime}$ is a uniform domain. Then there is a set $A \subset \partial G$ such that $A$ is dense in $\partial G$ and such that asy $(a, f) \neq \emptyset$ for every $a \in A$.

Proof. By II.3.10, there is $A \subset \partial G$ with $\bar{A}=\partial G$ such that each $a \in A$ is the endpoint of an endcut $\gamma_{a}$ which is a neargeodesic in $G$. By II.4.15, $f \gamma_{a}$ is a solid arc in $G^{\prime}$. Since $G^{\prime}$ is uniform, it follows from II.6.28 that $f \gamma_{a}$ is an endcut of $G^{\prime}$. Thus the point in $\overline{f \gamma}_{a} \cap \partial G^{\prime}$ is an asymptotic value of $f$ at $a$. व

3.3. Lemma. Suppose that $G \neq E$ is a domain and that $F$ is closed in $\dot{E}$ with $F \subset G$. If $k(\partial F) \leq c<\infty$, then $k(F) \leq c_{1}(c)<\infty$. gives

Proof. Let $a, b \in F$. Suppose first that $[a, b] \subset F$. Integration along $[a, b]$

$$
k(a, b) \leq \frac{|a-b|}{d(F, \partial G)} \leq \frac{d(F)}{d(F, \partial G)} .
$$

Since $F$ is bounded, we have $d(F)=d(\partial F)$. Since $d(F, \partial G)=d(\partial F, \partial G)$, we obtain by (2.6) and I.2.2

$$
k(a, b) \leq \bar{r}_{G}(\partial F) \leq 2\left(e^{c}-1\right) .
$$

Next assume that $[a, b] \not \subset F$. Choose $a_{1}, b_{1} \in[a, b] \cap \partial F$ such that $\left[a, a_{1}\right] \subset F$, $\left[b_{1}, b\right] \subset F$. Then

$$
k(a, b) \leq k\left(a, a_{1}\right)+k\left(a_{1}, b_{1}\right)+k\left(b_{1}, b\right) \leq 4\left(e^{c}-1\right)+c .
$$

Hence we can choose $c_{1}=4\left(e^{c}-1\right)+c$. ㅁ

3.4. Remark. It is natural to ask whether 3.2 holds with $c_{1}=c$. The following example shows that this is not the case: Let $E=R^{2}$ and let $n$ be a positive integer. Write $\alpha=2 \pi / n, r=\sin (\alpha / 2)$ and $x_{j}=e^{i j \alpha}$ for $j=0, \ldots, n-1$. 
Then $\left|x_{j-1}-x_{j}\right|=2 r$. We choose $n$ so large that $r<e^{-3 \pi}$. Next choose $R \geq 1+e^{\pi} / r$ and write

$$
\begin{aligned}
A & =\left\{x_{0}, \ldots, x_{n-1}\right\}, \\
G & =R^{2} \backslash A, \\
F & =\bar{B}(R) \backslash \bigcup_{j=0}^{n-1} B\left(x_{j}, r\right) .
\end{aligned}
$$

Then $F$ is compact and connected in $G$. Writing

$$
C=\bigcup_{j=0}^{n-1} S\left(x_{j}, r\right)
$$

we have $\partial F=S(R) \cup C$. Straightforward arguments based on I.2.2 show that

$$
k(F) \geq 2 \log \frac{1}{r}+\log (R-1) .
$$

Using suitable arcs joining points in $\partial F$ it is possible to show that

$$
k(\partial F) \leq \log \frac{1}{r}+\log (R-1)+3 \pi .
$$

Since $r<e^{-3 \pi}$, we see that $k(\partial F)<k(F)$.

However, the bound in 3.3 is probably far from the best possible. Indeed, I conjecture that one can choose $c_{1}=2 c$.

3.5. Phragmén-Brouwer properties. It is well known that for $n \geq 2$, the $n$ sphere $S^{n}$ has the Phragmén-Brouwer properties listed in [Wi, p. 47] or in [HY, p. 359]. It seems to be less known that several other spaces have these properties as well. We let $H_{p}(X)$ denote the integral singular homology groups of a space $X$. The key result is the following:

3.6. Theorem. Let $X$ be a topological space with $H_{1}(X)=0$. Suppose that $U$ and $V$ are pathwise connected open sets in $X$ with $U \cup V=X$. Then $U \cap V$ is pathwise connected.

Proof. We use the fact that a pair of points $a, b$ can be joined by a path in a set $A \subset X$ if and only if the singular 0-cycle $a-b$ represents the zero class in the reduced homology group $\tilde{H}_{0}(A)$. Since $U$ and $V$ are open, there is an exact reduced Mayer-Vietoris sequence

$$
\tilde{H}_{0}(U) \oplus \tilde{H}_{0}(V) \stackrel{\sigma}{\longleftarrow} \tilde{H}_{0}(U \cap V) \longleftarrow H_{1}(X) .
$$

Let $a, b \in U \cap V$, and let $\alpha \in \tilde{H}_{0}(U \cap V)$ be the element represented by $a-b$. Then $\alpha$ is mapped to zero by the homomorphisms induced by the inclusions $U \cap V \rightarrow U$ and $U \cap V \rightarrow V$. Hence $\sigma \alpha=0$. Since $H_{1}(X)=0$, it follows from exactness that $\alpha=0$. Hence $a$ and $b$ can be joined by a path in $U \cap V$. $\square$ 
3.7. Theorem. The one-point extension $\dot{E}$ of a Banach space $E$ with $\operatorname{dim} E \geq 2$ has all the Phragmén-Brouwer properties.

Proof. First observe that $\dot{E}$ is simply connected. Indeed, if $\operatorname{dim} E=n<\infty$, $E$ is homeomorphic to $S^{n}$. If $\operatorname{dim} E=\infty, \dot{E}$ is noncompact. Hence each loop $\alpha: S^{1} \rightarrow \dot{E}$ is nonsurjective and can therefore be deformed to the constant loop $\infty$.

It follows that $H_{1}(\dot{E})=0$. Moreover, $\dot{E}$ is connected and locally pathwise connected. By 3.6, $\dot{E}$ has the Phragmén-Brouwer property [Wi, Property I', p. 47]. Since $\dot{E}$ is metrizable, the theorem follows from [Wi, II.4.12]. व

3.8. Holes. Consider a domain $G \subset E$. A component of $\complement G=\dot{E} \backslash G$ is said to be a hole of $G$. The number of all holes of $G$ is written as $\nu(G)$. Then $\nu(G)$ is either a positive integer or $\infty$. By the following result, $\nu(G)$ is also the number of the boundary components of $G$ :

3.9. Theorem. If $C$ is a hole of $G$, then

(1) $\partial C=C \cap \bar{G}=C \cap \partial G \neq \emptyset$,

(2) $G \cup C$ is connected,

(3) $\complement C$ is connected,

(4) $C$ contains exactly one component of $\partial G$, namely $C \cap \bar{G}$.

Proof. Clearly

$$
C \cap \partial G=C \cap \bar{G} \subset \partial C \neq \emptyset .
$$

Suppose that there is $a \in \partial C \backslash(C \cap \bar{G})$. Then $a$ has a connected neighborhood $U \subset \complement G$. Now $C \cup U$ is a connected subset of $\complement G$ and strictly larger than $C$. This is impossible since $C$ is a hole, and (1) follows.

The property (2) is an obvious consequence of (1). Since $C C$ is the union of the sets $G \cup C^{\prime}$ over all holes $C^{\prime} \neq C$, we obtain (3).

Each component of $\partial G$ is contained in a hole of $G$. To prove (4) it suffices to show that $C \cap \bar{G}$ is connected. By (3), the set $G_{1}=\complement C$ is a domain with the single hole $C$. Using (1) twice we get

$$
C \cap \bar{G}=\partial C=C \cap \partial G_{1}=\partial G_{1} .
$$

By $3.7, \dot{E}$ has the Brouwer property [Wi, p. 47]. Hence $\partial G_{1}$ is connected. $\square$

3.10. Lemma. Suppose that $G \subset E$ is a domain and that $F \subset \dot{E}$ is a closed set which is the union of a family of holes of $G$. Suppose also that $U$ is a domain in $\dot{E}$ containing $F$. Then $U \backslash F$ is connected.

Proof. The open set $V=\complement F$ is the union of sets of the form $G \cup C$, where the sets $C$ are holes of $G$. From 3.9 (2) it follows that $V$ is connected. Moreover, $U \cup V=\dot{E}$. Since $U \backslash F=U \cap V$, the lemma follows from the Phragmén-Brouwer property of $\dot{E}$, given in 3.6 and 3.7. $\square$ 
3.11. Corollary. If $U$ is a domain in $\dot{E}$ with $\partial U \subset G$, then $U \cap G$ is a domain.

3.12. Definition. A set $F \subset \dot{E}$ separates the sets $A$ and $B$ if these sets are contained in different components of $\complement F$.

3.13. Theorem. Suppose that $f: G \rightarrow G^{\prime}$ is CQH. Suppose also that each pair of holes of $G$ can be separated by a set in $G$ of finite QH diameter. Then $\nu\left(G^{\prime}\right) \geq \nu(G)$.

Proof. Assume that $\nu\left(G^{\prime}\right)=s<\nu(G)$. Choose holes $C_{1}, \ldots, C_{s+1}$ of $G$. For each pair $1 \leq i<j \leq s+1$ we choose a set $F_{i j} \subset G$ with $k\left(F_{i j}\right)<\infty$ separating $C_{i}$ and $C_{j}$ in $\dot{E}$. Taking closures we may assume that each $F_{i j}$ is closed in $\dot{E}$. Let $F$ be the union of the sets $F_{i j}$. Then $F$ is closed in $\dot{E}, k(F)<\infty$, and the holes $C_{1}, \ldots, C_{s+1}$ lie in different components of $\complement F$. Since $f$ is $\mathrm{CQH}, k^{\prime}(f F)<\infty$, which implies that $f F$ is bounded and $d\left(f F, \partial G^{\prime}\right)>0$. Thus $f F$ is closed in $\dot{E}$.

Let $Q$ be a hole of $G^{\prime}$, and let $V_{Q}$ be the $Q$-component of $\complement f F$. Then $\partial V_{Q} \subset f F \subset G^{\prime}$. By 3.11, the open set $W_{Q}=V_{Q} \cap G^{\prime}$ is connected. Hence $f^{-1} W_{Q}$ is a domain in $G \backslash F$, and thus contained in a component of $\complement F$. Since $\nu\left(G^{\prime}\right)=s$, there is a component $U$ of $\complement F$ which contains a hole $C_{k}$ but does not meet $f^{-1} W_{Q}$ for any hole $Q$ of $G^{\prime}$.

Since $\partial U \subset F$, the set $D=U \cap G$ is a domain by 3.11. Let $U^{\prime}$ be the $f D$ component of $\complement f F$. If $U^{\prime}=V_{Q}$ for some hole $Q$ of $G^{\prime}$, then $D \subset f^{-1} W_{Q}$, which is impossible. Hence $U^{\prime} \subset G^{\prime}$. Moreover, $\bar{U}^{\prime} \subset U^{\prime} \cup f F \subset G^{\prime}$. Since $\partial \bar{U}^{\prime} \subset f F$, we have $k^{\prime}\left(\partial \bar{U}^{\prime}\right) \leq k^{\prime}(f F)<\infty$. By $3.3 k^{\prime}\left(\bar{U}^{\prime}\right)<\infty$, and hence $k^{\prime}(f D)<\infty$, which yields $k(D)<\infty$. This gives a contradiction, since $d(D, \partial G)=0$.

3.14. Remarks. 1. In the finite-dimensional case the condition of the theorem is always true.

2. We made use of the CQH property in the form $k(A)<\infty \Leftrightarrow k(f A)<\infty$. Hence the theorem holds for all homeomorphisms with this property.

3.15. Example. We construct a QH map $f: G \rightarrow G^{\prime}$ such that $\partial G$ is connected but $\partial G^{\prime}$ is not. Let $E$ be a separable Hilbert space. Combining a Möbius map and the map given in I.4.12 we obtain a $\mathrm{QH}$ map $g$ of the unit ball $B(1)$ onto a broken tube $D^{\prime}$ such that $\operatorname{clus}(e, g)=\operatorname{clus}(-e, g)=\emptyset$ for some $e \in \partial B(1)$. Set $G=B(1) \backslash[-e, e]$ and $G^{\prime}=f G$. Then $g$ defines a $\mathrm{QH}$ map $f: G \rightarrow G^{\prime}$. Now $\nu(G)=1, \nu\left(G^{\prime}\right)=2$.

3.16. Question. Does there exist a CQH map of a ball onto a domain with nonconnected boundary? 


\section{An example}

4.1. Introduction to Section 4. The most interesting examples of $\mathrm{QH}$ maps in infinite-dimensional spaces considered so far in these papers are the map onto a tube in I.4.11 and onto a broken tube in I.4.12. Moreover, the radial power map has been considered in I.4.10 and in I.6.5. In this section we give a variation of the tube map in a function space.

4.2. $L$-formulas. Suppose that $f: G \rightarrow E^{\prime}$ is a continuous map. Exceptionally, $E^{\prime}$ is allowed to be 1-dimensional. Recall from I.4.2 the notation

$$
L(x, f)=\limsup _{y \rightarrow x} \frac{|f y-f x|}{|y-x|} .
$$

The following formulas are easily verified:

$$
\begin{aligned}
L(x, f \pm g) & \leq L(x, f)+L(x, g), \\
L(x, \lambda f) & =|\lambda| L(x, f), \\
L(x, f g) & \leq L(x, f)|g x|+|f x| L(x, g), \\
L(x, f \circ g) & \leq L(g x, f) L(x, g) .
\end{aligned}
$$

They are valid whenever they make sense and contain no product of the form $0 \cdot \infty$ or $\infty \cdot 0$. In (3), one of the functions $f, g$ must be real-valued.

4.3. A function space. Suppose that $T$ is a compact Hausdorff space containing at least two points. Let $E=C(T)$ be the space of all continuous real-valued functions on $T$. Then $E$ is a Banach space with the norm

$$
\|x\|=\sup \{|x(t)|: t \in T\} .
$$

For $x \in E$ we let $M(x)$ and $m(x)$ denote the maximum and minimum of $x$, and we write osc $x=M(x)-m(x)$.

4.4. The map $f$. The sets

$$
G=\{x: m(x)>0\}, \quad G^{\prime}=\{x: \operatorname{osc} x<1\}
$$

are clearly domains in $E$. Alternatively,

$$
G^{\prime}=\{x: d(x, \Delta)<1 / 2\}
$$

where $\Delta$ is the line of all constant functions. We define a map $f: G \rightarrow G^{\prime}$ by

$$
f(x)=\frac{x}{M(x)}+\log M(x)=\frac{x}{\|x\|}+\log \|x\|,
$$


or more precisely,

$$
f(x)(t)=\frac{x(t)}{M(x)}+\log M(x) .
$$

To verify that $f(x)$ is in $G^{\prime}$ observe that

$$
M(f(x))=1+\log M(x), \quad m(f(x))=m(x) / M(x)+\log M(x),
$$

and hence

$$
\text { osc } f(x)=1-m(x) / M(x)<1 .
$$
phism.

4.5. Theorem. The map $f: G \rightarrow G^{\prime}$ defined in 4.4 is a 6 -QH homeomor-

Proof. We first define a map $g: G^{\prime} \rightarrow E$ by

$$
g(y)=e^{M(y)-1}(y-M(y)+1) .
$$

Since

$$
m(g(y))=e^{M(y)-1}(1-\text { osc } y)>0,
$$

$g(y) \in G$. It is easy to verify that $f \circ g=$ id and $g \circ f=$ id. Since $f$ and $g$ are clearly continuous, $f$ is a homeomorphism with $f^{-1}=g$.

It is easy to see that

$$
\partial G=\{x: m(x)=0\} \cup\{\infty\}, \quad \partial G^{\prime}=\{x: \operatorname{osc} x=1\} \cup\{\infty\} .
$$

Furthermore,

$$
\delta(x)=m(x), \quad \delta^{\prime}(y)=(1-\operatorname{osc} y) / 2
$$

for $x \in G, y \in G^{\prime}$. The first equality is obvious, and we prove the second one. Let $y \in G^{\prime}$ and write $\alpha=1-\operatorname{osc} y>0$. Assume that $z \in \partial G^{\prime}, z \neq \infty$. Since osc $z=1$, we have either $M(z) \geq M(y)+\alpha / 2$ or $m(z) \leq m(y)-\alpha / 2$. Hence $\|y-z\| \geq \alpha / 2$, which implies $\delta^{\prime}(y) \geq \alpha / 2$. To prove that $\delta^{\prime}(y) \leq \alpha / 2$ we construct $z \in \partial G^{\prime}$ with $\|z-y\|=\alpha / 2$. If $y$ is a constant function $y(t)=c$, we use Uryson's lemma to find $z \in E$ with $M(z)=c+1 / 2, m(z)=c-1 / 2$. Then $z \in \partial G^{\prime}$ and $\|z-y\|=1 / 2=\alpha / 2$. If $y$ is not constant, let

$$
v:[m(y), M(y)] \rightarrow[m(y)-\alpha / 2, M(y)+\alpha / 2]
$$

be the affine increasing homeomorphism. Then $z=v \circ y \in \partial G^{\prime}$, and $\|z-y\|=\alpha / 2$.

To prove that $f: G \rightarrow G^{\prime}$ is 6-Lipschitz in the QH metric it suffices to show, by I.4.6, that

$$
L(x, f) \delta(x) \leq 6 \delta^{\prime}(f x)
$$


at an arbitrary point $x \in G$. We obviously have $L(x, M) \leq 1$ and $L(x$,id $)=1$. Writing $h(x)=1 / M(x)$ we obtain from the $L$-formulas in 4.2

$$
\begin{aligned}
& L(x, h) \leq 1 / M(x)^{2}, \\
& L(x, f) \leq L(x, h) M(x)+h(x) L(x, \text { id })+L(x, M) / M(x) \leq 3 / M(x) .
\end{aligned}
$$

Since (4.6) gives

$$
\delta^{\prime}(f x)=\frac{1-\operatorname{osc} f(x)}{2}=\frac{m(x)}{2 M(x)}
$$

we obtain (4.7).

It remains to show that $g$ is 6-Lipschitz in the QH metric. Let $y \in G^{\prime}$. Then $0<y-M(y)+1 \leq 1$. Using the formulas of 4.2 we obtain

$$
\begin{aligned}
L(y, g) & =e^{M(y)-1} L(y, M)(y-M(y)+1)+e^{M(y)-1}(L(y, \mathrm{id})+L(y, M)) \\
& \leq 3 e^{M(y)-1} .
\end{aligned}
$$

By (4.6) we have

$$
\delta(g y)=m(g y)=e^{M(y)-1}(1-\operatorname{osc} y)
$$

and thus

$$
L(y, g) \delta^{\prime}(y) \leq 3 e^{M(y)-1}(1-\text { osc } y) / 2=3 \delta(g y) / 2 .
$$

Hence $g$ is indeed (3/2)-Lipschitz in the QH metric. $\square$

4.8. Remark. Consider the special case of the example above where $T$ consists of two points. Then $E$ is the plane $R^{2}$ with the norm $\|x\|=\left|x_{1}\right| \vee\left|x_{2}\right|$, $G$ is the quarter plane $x_{1}>0, x_{2}>0$, and $G^{\prime}$ is the parallel strip $\left|x_{1}-x_{2}\right|<1$.

\section{References}

[HY] Hocking, J.G., and G.S. Young: Topology. - Addison-Wesley, 1961.

[Kl] KLEE, V.L.: A note on topological properties of normed linear spaces. - Proc. Amer. Math. Soc. 7, 1956, 673-674

[TV] Tukia, P., and J. VÄISÄLÄ: Lipschitz and quasiconformal approximation and extension. - Ann. Acad. Sci. Fenn. Ser. A I Math. 6, 1981, 303-342.

[Vä ${ }_{1}$ ] VÄIsÄL $\ddot{A}$, J.: Free quasiconformality in Banach spaces I. - Ann. Acad. Sci. Fenn. Ser. A I Math. 15, 1990, 355-379.

[Väㄱ ${ }_{2}$ VÄıs̈̈L̈̈, J.: Free quasiconformality in Banach spaces II. - Ann. Acad. Sci. Fenn. Ser. A I Math. 16, 1991, 255-310.

[Vu] Vuorinen, M.: Capacity densities and angular limits of quasiregular mappings. - Trans. Amer. Math. Soc. 263, 1981, 343-354.

[Wi] Wilder, R.L.: Topology of manifolds. - Amer. Math. Soc. Colloquium Publications 32, 1949. 